\title{
Propiedades Fisicoquímicas De Suelos Cultivados Con Asparagus Officinalis En La Región Árida Del Noroeste De México
}

\author{
Rosa A. Vázquez, M.C. \\ Luis Manuel Lozano Cota, Dr. \\ Lamberto Castro Arce, Dr. \\ Ramona Icedo García, Dr.
}

Departamento de Cs. Químico Biológicas y Agropecuarias de la

Universidad de Sonora, Unidad Regional Sur

Beatriz E. Arias-T, Dr.

Departamento de Cs. Químico Biológicas y Agropecuarias de la

Universidad de Sonora, Unidad Regional Norte

Edgar Omar Rueda-Puente, Dr.

Universidad de Sonora, Departamento de Agricultura y Ganadería, Carretera a Bahía de Kino km. 21. Hermosillo, Sonora, México

\begin{abstract}
In the agriculture of arid zones, the inadequate soil management has generated changes in their quality and sustainability, affecting the structure and physicochemical conditions which reduce crop yields; based on the above, is consider as a vital importance the analyses of soil and plants because they provide information, current and accurate, which can be useful to take decisions in relation to soil fertility and plant nutrition. The inappropriate handling and amount of fertilizers in asparagus in the arid region of the Northwest of Mexico, has caused low yields. The aim of this study consisted to analyze soil in seven of the thirteen sub regions of the agricultural coast of Caborca, Sonora, where the asparagus crop has been planted for more than 10 years. The analyzes performed were: percentage of saturation, $\mathrm{pH}$, electrical conductivity (CE), soil texture Nitrogen $\left(\mathrm{NO}_{3}\right), \mathrm{P}$, $\mathrm{K}, \mathrm{Na}, \mathrm{Ca}, \mathrm{Mg}$ and cation exchange capacity. The results showed high levels of Nitrogen and Potassium in almost all the studied sites; on contrary Phosphorus, Calcium and Magnesium, presented values below of the recommended and Electrical conductivity above 5.5. mmhos/ $\mathrm{cm}$. The integration of this information allows appropriate technical advice, to achieve the balance of nutrients, such as to avoid unnecessary costs in
\end{abstract}


fertilizers that the plant might can not take, in addition to the resulting contamination of soil to leach these products.

Keywords: nutrition, esparrago, yield production, salinity.

\section{Resumen}

En la agricultura de las zonas áridas, el manejo inadecuado de suelos ha generado alteraciones en su calidad y sostenibilidad, afectando su estructura y condiciones fisicoquímicas que reduce la producción de los cultivos; con base a lo anterior, se consideran de importancia los análisis de suelos y plantas ya que proporcionan información, actual y precisa, que puede ser de utilidad para la toma de decisiones en relación con a la fertilidad del suelo y con la nutrición de la planta. El inapropiado manejo y cantidad de fertilizantes en espárrago en la región árida del Noroeste de México, ha ocasionado que los rendimientos sean bajos. El objetivo del presente estudio consistió en analizar suelos en siete de las trece subregiones de la Costa Agrícola de Caborca, Sonora, donde el cultivo de espárrago, se ha sembrado por más de 10 años. Los análisis que se realizaron fueron: Porcentaje de saturación, pH, Conductividad eléctrica (C.E.), Textura, Nitrógeno $\left(\mathrm{NO}_{3}\right)$, Fósforo, Potasio, Sodio, Calcio, Magnesio y Capacidad de Intercambio Catiónico. Los resultados de estos análisis muestran niveles altos de Nitrógeno y Potasio en la mayoría de los sitios estudiados; por el contrario, el Fósforo, Calcio y Magnesio, presentaron valores por debajo de lo recomendado y Conductividades eléctricas arriba de $5.5 \mathrm{mmhos} / \mathrm{cm}$. La integración de ésta información permite hacer las recomendaciones técnicas apropiadas, tanto para lograr el balance de nutrientes, como para evitar gastos innecesarios en fertilizantes que la planta no puede aprovechar, además de la consecuente contaminación del suelo al lixiviarse estos productos.

Palabras clave: Nutrición, espárrago, producción, salinidad

\section{Introducción}

Cerca del 50\% del total de los suelos irrigados en el mundo están afectados por la salinización y/o alcalinización, lo cual se asocia a baja productividad de cultivos convencionales (Rueda et al., 2009). Los suelos salinos se encuentran principalmente en zonas de clima árido o semiárido. En este tipo de regiones, las sales solubles no pueden ser transportadas muy lejos, esto ocurre no solamente porque hay menor precipitación para lavar y transportar las sales, sino también a consecuencia de la elevada evaporación característica del clima árido, que tiende a concentrar las sales en los suelos y en el agua superficial (Aceves, 1979). Para utilizar tierras con altas 
concentraciones de sales, algunos científicos se han abocado al desarrollo de cultivos tolerantes a la sal o halófitos (Rueda et al., 2003). Algunas especies de semi-halófitas, presentan un potencial económico por su alta productividad para la agricultura del desierto y zona costera o bien en aquellas áreas que no están muy salinizadas. Diversos propósitos tienen las halófitas, entre los que figuran alimento humano, forraje, cosméticos, extractos con uso biopesticida, biodiesel, etc (Rueda et al., 2009). A nivel mundial, los ecofisiólogos están orientando los esfuerzos de investigación para generar tecnologías agrícolas que innoven los cultivos agroindustriales o bien desarrollar recursos vegetales endémicos que posean alto potencial de agro-industrialización, y que gracias a su capacidad de producción en suelos salinos o condiciones de sequía, permitan optimizar la productividad del campo mexicano (Reig, 1994). Las zonas áridas producen el $60 \%$ de los alimentos a nivel mundial (De Santa Olalla et al., 2005). Una de las zonas de relevancia en la producción de alimentos en la República Mexicana, es el noroeste de México, el cual está conformado por los estados de Baja California, Baja California Sur, Chihuahua, Sinaloa y Sonora. Las condiciones que prevalecen en esta región van desde los 50 a $230 \mathrm{~mm}$ de precipitación anual, con temperaturas que oscilan en los meses de verano entre los 38 a los $52^{\circ} \mathrm{C}$; una marcada fluctuación de temperaturas diurna/ nocturna y de la velocidad del viento; baja disponibilidad de agua, debido a la alta evaporación; una radiación solar incidente; los suelos de estas zonas son variables en profundidad, textura, $\mathrm{pH}$ alcalinos, conductividad eléctrica altas y baja fertilidad; en ellos se acumulan carbonato y la tasa de infiltración del horizonte cálcico depende del contenido de carbonato (López- Aguilar et al., 2008); La intrusión salina se enfatiza provocando que las conductividades eléctricas varíen entre los 2 a los 10 decisiemens, ensalitrando los pozos de agua utilizados para la agricultura y que lleguan a contener hasta $8 \pm 4$ grL de agua con sólidos disueltos (Rueda et al., 2009).

Con base en la problemática descrita para la agricultura en las zonas áridas, los análisis de suelos y plantas proporcionan información importante, actual y precisa que es de utilidad para la toma de decisiones relacionadas con la fertilidad del suelo y la nutrición de la planta. De los análisis de suelos depende el lograr los máximos beneficios en producción.

En el noroeste de México, zona árida significativa en la producción de alimentos, la región de Caborca, Sonora, posee suelos jóvenes y profundos, predominando los Aridisoles y Entisoles, con texturas arenosas y francas, pobres en materia orgánica, baja capacidad de retención de humedad, presencia de carbonato de calcio y potencial de hidrógeno $(\mathrm{pH})$ alcalinos. Un cultivo semitolerante a la salinidad es el del espárrago (Asparagus officinalis); existen dos limitantes importantes en la productividad de sus plantaciones: a) los factores de crecimiento (riego y 
nutrición) y b) las condiciones ambientales presentes en la región (radiación solar intensa, ciclo de crecimiento largo y baja nubosidad). En referencia a la primera limitante, los productores y técnicos con la finalidad de incrementar sus rendimientos acuden a altas dosificaciones de fertilizantes químicos, lo cual conlleva a agudizar el problema de la salinidad en suelos, repercutir negativamente en la microflora presente en los suelos, incrementando los pH's (Aceves, 1979); además de que las pantas de espárrago crezcan de forma exuberante, provocando rebrotes durante el ciclo de crecimiento, afectando directamente los costos de producción, así como la productividad y la calidad del espárrago (Cabrera, 1993). Con base en z lo anterior, el objetivo de la presente investigación consistió en evaluar los parámetros fisicoquímicos de suelos sembrados con espárrago, cultivo que lleva más de 10 años en la región de Caborca, Sonora, México.

\section{Material y Métodos:}

\section{Selección del sitio de estudio}

Con el fin de hacer representativo el muestreo de toda el área productora de espárrago, se tomó como referencia el mapa del Distrito de Desarrollo Rural No. 139 Caborca, el cual se localiza en el paralelo 3042' de latitud norte y el meridiano $112^{\circ} 09^{\prime}$ de longitud al oeste de Greenwich y a una altura de 289 metros sobre el nivel del mar, del cual se seleccionaron únicamente siete de las trece subregiones de la Costa Agrícola de Caborca. Con fines de selección del sitio se establecieron los criterios siguientes: 1) plantaciones con riego presurizado y 2) plantaciones en plena producción. En el Cuadro 1 se muestran las subregiones seleccionadas para el estudio, que-se denominaran como sitios de estudio. Una vez seleccionados los sitios en cada una de las subregiones, se iniciaron los muestreos en el mes de marzo y se concluyeron en el mes de abril, un año después. Diez muestreos fueron realizados con un espacio de cincuenta días entre cada uno, recolectándose tres muestras de suelo (tres repeticiones) por sitio de estudio.

\section{Colección de la muestra}

La profundidad de muestreo fue entre $30-40 \mathrm{~cm}$ ya que corresponde al lugar en el que crecen la mayor parte (85\%) de las raíces de espárrago y es el espacio donde está la absorción de agua y nutrientes; las muestras de 1 kg aproximado de suelo fueron depositadas en bolsas de plástico de color negras.

\section{Preparación de las muestras de suelo}

El material, equipo y procedimiento para la preparación de la muestra incluyó el traslado hacia el laboratorio en recipientes sellados con geles anticongelantes, etiquetado de muestras de sitio, registro en laboratorio, 
secado de muestras de suelo a la intemperie (con temperaturas de $47^{\circ} \mathrm{C} \pm 3$, por 24 horas, extendiéndolas sobre una superficie de papel periódico, molienda, tamizado y almacenamiento (Licon et al., 2001).

\section{Análisis fisicoquímicos y métodos de análisis utilizados en el laboratorio}

A cada muestra de suelo se les realizaron análisis físicos: \% de saturación, $\mathrm{pH}$, conductividad eléctrica y textura, así como análisis químicos: Concentración de nitrógeno $\left(\mathrm{NO}_{3}\right)$, fósforo, calcio, magnesio, sodio y potasio. Dichos análisis se llevaron a cabo en el Laboratorio de suelos de la Universidad de Sonora, Unidad Regional Norte campus Caborca. En el Cuadro 2 se describen los métodos de análisis de laboratorio utilizados (Aguilar et al., 1987).

Para determinar la textura del suelo se utilizó un Hidrómetro de Bouyoucos número 1,524 con escala en gramos por litro, mezclador eléctrico, cilindro de sedimentación con aforo de 1130 a 1205, probeta de 10 ml, termómetro en grados centígrados y como reactivos se requirió de Solución de Calgón, disolviendo 38.7 g de Hexametafosfato de sodio y 7.94 g de carbonato de sodio en 1 litro de agua. Una vez obtenidos los datos de porcentajes, se buscó en el triángulo de textura el punto de intersección, obteniendo de esta manera la clasificación de textura correspondiente.

Para la determinación del \% de saturación, primero se preparó una pasta saturada que requirió recipientes de plástico de $500 \mathrm{ml}$, bureta graduada de $500 \mathrm{ml}$ o de $1000 \mathrm{ml}$, balanza granataria y una espátula plana. El procedimiento consistió en 1) pesar 400 g de suelo, 2) pasarlos a un recipiente de plástico de preferencia con tapadera, 3) agregar agua al suelo utilizando la bureta de 500 o 1000 ml, 4) mezclar constantemente con una espátula plana hasta llegar al punto de saturación 5) anotar los mililitros de agua gastados (Ruíz et al., 1979).

Para medir el $\mathrm{pH}$ del suelo en pasta saturada, se utilizó un potenciómetro marca Fisher Scientific para lo cual se procedió a introducir cuidadosamente el electrodo del potenciómetro dentro de la pasta saturada anteriormente y se determinó el $\mathrm{pH}$ de acuerdo con las instrucciones del aparato utilizado (Pérez y Werner, 2007).

Para la determinación de la Conductividad eléctrica (CE), se hizo uso de un puente de Conductividad marca Corning modelo Pinnacle 54. Los reactivos utilizados fueron; cloruro de potasio $0.01 \mathrm{~N}$ para lo cual se necesitó disolver $0.7456 \mathrm{~g}$ de $\mathrm{KCl}$ en agua destilada y aforar a 1 litro. La conductividad de esta solución es de $0.0014118 \mathrm{mmhos} / \mathrm{cm}$ a $25{ }^{\circ} \mathrm{C}$. El procedimiento consistió en 1) calibrar el equipo con el estándar conocido 2) enjuagar la celda con agua destilada y llenar con el extracto procurando que no queden burbujas 3) colocar la muestra para que el electrodo reporte la 
lectura 4) retirar el extracto y enjuagar 2- 3 veces con agua destilada para continuar con la siguiente muestra (Ruíz et al., 1979).

Para analizar el nitrógeno $\left(\mathrm{NO}_{3}\right)$ se utilizó un equipo Espectronic 20. El procedimiento consistió en: 1) Pesar 10 g de suelo seco y tamizado con la malla de $2 \mathrm{~mm}$ y se coloca en un matraz Erlenmeyer de $125 \mathrm{ml}, 2$ ) agregar $50 \mathrm{ml}$ de solución extractora volumen inicial (Vi), 3) agitar durante 10 minutos en el agitador mecánico, 4) filtrar en un vaso precipitado con el papel filtro, en caso de turbidez se filtró por segunda vez, 5) tomar $10 \mathrm{ml}$ del filtrado, colocarlo en un vaso precipitado de $100 \mathrm{ml}$ y ponerlo en la estufa hasta que se evapore completamente a una temperatura de $80{ }^{\circ} \mathrm{C}$, 6) sacar la muestra de la estufa y dejar que se enfríe a temperatura ambiente, 7) agregar $2 \mathrm{ml}$ de ácido fenoldisulfónico tratando de que todo el ácido entre en contacto con todos los residuos, dejar reposar 5 minutos, agregar de 30 a 40 $\mathrm{ml}$ de agua destilada y $20 \mathrm{ml}$ de hidróxido de amonio al 50\%, 8) pasar la solución a un matraz volumétrico de 100, aforar a un volumen final (Vf) con agua destilada y agitar, 9) tomar lectura en el espectrofotómetro a una longitud de onda de 420 nanómetros calibrado con un blanco de reactivo o con agua destilada. 10) anotar el \% de transmitancia y compararlo con la curva patrón (Santelises et al., 1987).

Para la determinación de fósforo en suelo se utilizó un Espectronic 20 y como reactivos se contó con Solución extractora Bray P-1, cloruro estañoso diluido, molibdato de amonio y agua destilada. El procedimiento para realizar este análisis consistió en: 1) pesar 5 g de suelo seco y tamizado 2) agregar $35 \mathrm{ml}$ de solución extractora BRAY P1 3) agitar manualmente 2 minutos, 4) filtrar con papel Whatman No. 42, 5) tomar $2 \mathrm{ml}$ del filtrado 6) agregar $5 \mathrm{ml}$ de agua destilada 7) agregar $2 \mathrm{ml}$ de molibdato de amonio y se mezclan bien, 8) agregar $1 \mathrm{ml}$ de cloruro estañoso diluido. (Se prepara al momento de su uso ya que tiene una vida útil de 2 horas) 9) tomar la lectura de 5 - 20 minutos después de haber agregado el cloruro estañoso y preferiblemente 10 min después, 10) leer \% de transmitancia en el espectrofotómetro a $660 \mathrm{~nm}$, ajuste colorímetro con un blanco reactivo (Aguilar et al., 1987).

Para conocer el contenido de macroelementos en suelo ( $\mathrm{Na}$, K, Ca y $\mathrm{Mg}$ ), se requirió contar con un equipo de absorción atómica, en este caso se utilizó un AA marca GBC 932 Plus. El material utilizado fue: matraces Erlenmeyer de $125 \mathrm{ml}$, vasos de precipitado de $100 \mathrm{ml}$, matraces volumétricos de 25 y $50 \mathrm{ml}$, pipetas de 1 y $5 \mathrm{ml}$, agitador mecánico y papel filtro Wathman 42. Los reactivos requeridos fueron: Acetato de Amonio, Cloruro de Litio y Cloruro de Lantano. El procedimiento consistió en: 1) pesar $2.5 \mathrm{~g}$ de suelo seco y tamizado y pasarlo a un matraz Erlenmeyer de $125 \mathrm{ml}, 2$ ) agregar $25 \mathrm{ml}$ de acetato de amonio 3) agitar por espacio de media hora en agitador mecánico, 4) filtrar con papel filtro. Para analizar Na, K y 
Mg, se tomaron $1 \mathrm{ml}$ del filtrado en un matraz volumétrico de $50 \mathrm{ml}$; se agregan $5 \mathrm{ml}$ de Cloruro de Litio y aforar a la marca; para analizar (Ca), se toman $1 \mathrm{ml}$ del filtrado en un matraz volumétrico de $25 \mathrm{ml}$; se agregan 2.5 $\mathrm{ml}$ de Cloruro de Lantano y se afora a la marca; posteriormente se procede a realizar las lecturas en el espectrofotómetro de absorción atómica con las especificaciones de cada elemento (Aguilar et al., 1987).

\section{Resultados}

\section{Textura}

Los resultados indican que en los sitios I, II, III, V y VI los suelos fueron textura areno-francoso (Cuadro 5, 6, 7, 8, 9, 10); para el caso del sitio IV la textura resultó franco-arenoso y en el sitio VI la textura fue arenosa. Estos suelos tienen alto porcentaje de arena (65.58 - 93.58\%), limo (4.06 $19.06 \%$ ) y $3.36-15.36 \%$ de arcilla. Los resultados anteriores confirman los datos reportados por la Secretaría de Recursos Hidráulicos (SARH, 1987), donde se señala que los suelos que predominan en el Distrito de Desarrollo Rural No. 139 Caborca, son suelos profundos, de texturas arenosas y francas. La mayoría de la literatura relacionada con tecnología de producción de espárrago, indica que el suelo más aconsejable para cultivarlo es el de textura franco arenosos a arenoso profundo por las ventajas que presentan tales como: la labranza es menos laboriosa y de menor costo en suelos arenosos que son bien aireados, secan rápido y no forman costra en la superficie dando como resultado un producto del esparrago más atractivo que en suelos pesados. Sin embargo, no es común que el suelo sea demasiado suelto o ligero, de modo especial si el subsuelo está compuesto por arena y grava, debe de tomarse en cuenta que el subsuelo debe retener humedad manteniendo un buen drenaje; por otra parte, los suelos arenosos, aunque tienen textura deseable para la buena emergencia del turión, alcanzan temperaturas de superficie muy elevadas que pueden afectar la calidad del mismo (Souther et al., 1986; Gardner y Roth, 1989; Orduño, 2004). Basados en esta información el tipo de textura del suelo en la región Agrícola de Caborca, es de los más adecuados para cultivar espárrago.

\section{Potencial de hidrógeno (pH)}

El pH de la solución del suelo más deseable para espárrago debe estar entre 6.5 y 7.5, condiciones ácidas $\mathrm{pH}<$ de 6.5 puede llevar a reducir crecimiento y por consecuencia el rendimiento. Los valores de $\mathrm{pH}$ de los sitios identificados en el presente estudio fluctúan entre 7.2 y 7.5 , lo que significa que los suelos en los que se cultiva el espárrago en Caborca presentan el $\mathrm{pH}$ adecuado para el cultivo, ya que la mayoría de los elementos minerales se encuentren disponibles para ser absorbidos, lo cual coincide con lo encontrado estudios previos (Mullen y Laemlen, 2008). 


\section{Conductividad eléctrica (CE)}

En relación a salinidad, se encontró que la conductividad eléctrica de los suelos estudiados, fluctuó en un rango de 1.5 a $9.7 \mathrm{mmhos} / \mathrm{cm}$. Estos resultados indican que el espárrago en esta región puede crecer y desarrollarse, pero posiblemente pueden presentarse algunos daños aparentes, esto dependerá del tipo de suelo y-calidad de agua de riego (Sánchez, 2006). El espárrago es una hortaliza que soporta altas concentraciones de salinidad ya que es capaz de crecer adecuadamente en el rango de 5 a 8 mmhos/cm (Ayres y Westcot, 1985). Sánchez (2006), reporta umbrales para este cultivo de 4.1 hasta $10 \mathrm{mmhos} / \mathrm{cm}$, señalando que éstos varían de acuerdo a las condiciones de los diferentes lugares en los que se cultiva el espárrago. De acuerdo a resultados de investigación en relación a la CE se reporta que dentro de un rango de 1.5 - $2.5 \mathrm{mmhos} / \mathrm{cm}$ el follaje del espárrago se observa completamente sano y verde, pero a medida que la CE va aumentado y alcanza un valor arriba de $2.5 \mathrm{mmhos} / \mathrm{cm}$, las hojas van mostrando daño de quemaduras casi hasta la mitad de la hoja, consecuentemente la biomasa disminuye debido a que afecta directamente la superficie fotosintética y como resultado se ven afectados los rendimientos (Francois, 1987).

\section{Nitrógeno $\left(\mathrm{NO}_{3}\right)$}

Los resultados obtenidos muestran fluctuación en la concentración de $\mathrm{NO}_{3}$ en el suelo durante la estación de crecimiento en los diferentes sitios experimentales (Cuadro 5, 6, 7, 8, 9, 10). Las diferencias encontradas posiblemente se deban a varios factores, entre los que se encuentran: a) el tipo de suelo b) manejo del agua, c) edad de las plantaciones, d) plan de fertilización en cuanto a dosis y fechas e) fuente de fertilizante que se utiliza en cada sitio y f) la diferencia en $\mathrm{pH}$ y conductividad eléctrica. El sitio V que tiene 19 años, tendió a mostrar los valores más altos de concentración de nitratos, mientras que el sitio IV que tiene 12 años presentó los valores más bajos de $\mathrm{NO}_{3}$. La diferencia en concentración entre el sitio $\mathrm{V}$ y el sitio IV fue de $29 \mathrm{ppm}$ en promedio en todo el ciclo de crecimiento, esto se debe probablemente a que se aplicó más nitrógeno de acuerdo a la edad de la plantación en el sitio V que en el sitio IV.

\section{Fósforo (P)}

La concentración de fósforo mostró una fluctuación similar a la del nitrógeno en cuanto a los fuertes incrementos y descensos, sin embargo las concentraciones de fósforo en suelo entre los distintos sitios fueron mínimas (Cuadro 5, 6, 7, 8, 9, 10). Se observó que la demanda es constante durante todo el período, lo cual tiene relación con la asimilación que está llevando a cabo la planta ya que interviene en la formación de raíces y esto ocurre 
durante la mayor parte del ciclo de crecimiento, participa también en la transferencia de energía dentro del tejido celular y en la composición de cromosomas, ADN y ARN (Porta et al., 1999).

\section{Potasio (K)}

El potasio mostró una dinámica más parecida a la observada con el nitrógeno que con el fósforo (Cuadro 5, 6, 7, 8, 9, 10); ya que las concentraciones en el suelo de este elemento fueron altas y durante el ciclo de desarrollo del cultivo se observaron fuertes incrementos seguidos de bruscos descensos, aunque los valores en partes por millón fluctúan o varían entre sitios, obteniendo valores de 3000 ppm más alto el sitio V y menores como 500 ppm en el sitio VI, lo que básicamente se puede deber a que se aplicaron cantidades distintas considerando las particularidades en cada sitio. De acuerdo a observaciones de campo de varios asesores técnicos de espárrago para la región de Caborca, se reporta que la presencia de potasio en los suelos cuando se cultiva esta hortaliza es vital al final del ciclo, especialmente a finales de agosto y septiembre ya que se requiere para que madure bien el cultivo, descanse y tenga una buena dormancia; situación que coincide con Ferreyra et al. (1995).

\section{Magnesio (Mg)}

Una de las características del magnesio es que presentó un comportamiento diferente a $\mathrm{N}, \mathrm{P}$ y $\mathrm{K}$, ya que sólo al inicio del ciclo de crecimiento hay aumento en las concentraciones (Cuadro 5, 6, 7, 8, 9, 10), esto probablemente se debe a que la mayoría de los productores de espárrago en la región hacen aplicaciones de este nutriente al principio del ciclo; en el resto del ciclo este elemento se presenta más estable. La tendencia en los sitios fue similar, conforme va creciendo la planta los requerimientos van aumentando. En noviembre y enero es menor el incremento.

\section{Calcio (Ca)}

La concentración de calcio tuvo un aumento al inicio del ciclo manteniéndose estable el resto del mismo (Cuadro 5, 6, 7, 8, 9, 10), esto puede deberse a que se hacen las aplicación totales al principio, cuando empieza el ciclo, se mantiene estable durante el ciclo de crecimiento de la planta, significando una demanda paulatina pero constante; sin embargo, al final del ciclo se observa un descenso en la mayoría de los sitios, lo cual indica que la planta lo requiere al momento de la cosecha porque es utilizado junto con el potasio ya que deben ir balanceados.

\section{Niveles de referencia de nutrientes en suelo y concentraciones de nitrógeno $\left(\mathrm{NO}_{3}\right)$, fósforo y potasio detectados}


Con el fin de realizar un diagnóstico general de las concentraciones en las que se encuentran los nutrientes en el suelo, se tomaron en cuenta los Estándares de referencia de los laboratorios de suelos en México en relación a los nutrientes en suelo para cualquier cultivo (Pérez y Werner, 2007), los cuales se muestran en el Cuadro 3.

Para nitratos $\left(\mathrm{NO}_{3}\right)$, se reporta como nivel de referencia $30 \mathrm{ppm}$ sin embargo, los resultados obtenidos muestran que en la mayoría de los suelos estudiados se encuentran por encima de ese nivel (Cuadro 5, 6, 7, 8, 9, 10), esto puede significar que se fertiliza en forma excesiva con ese nutriente. En el caso del fósforo, los resultados muestran que todos los suelos estudiados están por debajo de esos niveles recomendados. Si se toma en cuenta que el fósforo debe estar en una concentración de 30 ppm en el suelo. Montes y Holle, 1978 y Espejo et al. (1997), establecen que para espárrago se manejan 20 ppm como niveles necesarios para que el elemento cumpla sus funciones en la planta, esto significó que en 6 sitios del estudio, este elemento estuvo en el nivel adecuado. Para el caso del sitio que mostró bajos niveles de fósforo puede suponerse que se haya dado la importancia debida en la fertilización. Respecto al potasio, los resultados muestran que en la mayoría de los casos se encuentra por encima de los niveles adecuados (Cuadro 5, 6, $7,8,9,10$ ), esto indica que el productor se preocupa por tener mucho potasio en el suelo para que a la planta no le haga falta en las diferentes etapas del ciclo, es por ello que fertiliza continuamente de tal manera que el elemento se encuentra siempre disponible. Sin embargo, esto representa un gasto innecesario para el productor ya que es un fertilizante que no será aprovechado por la planta, en cambio va quedar fijado al suelo al igual que al fósforo.

Por otro lado, los niveles de calcio encontrados están por debajo del nivel adecuado en la mayoría de los casos (Cuadro 5, 6, 7, 8, 9, 10); sin embargo, por las apreciaciones visuales en todas las plantaciones de espárrago estudiadas no se vieron síntomas de deficiencia de este elemento tales como hojas terminales cloróticas y con coloración ceniza (Sánchez, 2005). Aún y cuando se observaron niveles bajos del elemento, según los técnicos de la región no sería recomendable hacer aplicaciones, ya que se debe tomar muy en cuenta que las aguas de riego en esta zona tienen concentraciones altas de carbonatos y bicarbonatos que pueden precipitarse como carbonatos de calcio. Cuando se presentan acumulaciones de carbonato a cierta profundidad en el perfil del suelo, las plantas pueden sufrir la muerte de su yema apical, después de haber tenido un desarrollo inicial normal (Aceves, 1979; Núñez y Casas, 2000). Para el magnesio se encontraron resultados similares a los del calcio (Cuadro 5, 6, 7, 8, 9, 10), ya que en la mayoría de los suelos se encuentra que el magnesio está por debajo de los 
niveles adecuados, excepto en dos sitios, el resto se encuentran cercanos al nivel adecuado.

En el caso del sodio, los resultados muestran que sus concentraciones se encuentran por encima de los niveles adecuados (Cuadro 5, 6, 7, 8, 9, 10). De acuerdo a Navarro (2002), indica que durante varios ciclos, en muchas de las plantaciones de espárrago en la región, en verdores o brotaciones que cumplen su ciclo (follaje viejo) se logra ver mitades de hojas con sintomatología de exceso de sodio como hojas necróticas generadas por la sales de sodio. Estos resultados permiten sugerir al productor la importancia de realizar prácticas de mejoramiento de suelo principalmente en aquellas plantaciones con riego por goteo para disminuir el contenido de sodio en el suelo; igualmente importante sería estudiar las condiciones de calidad de su agua de riego ya que existen reportes que mencionan que el contenido de sodio en el agua también es un factor que se debe tomar en cuenta ya que el sodio puede llegar a desplazar a iones de calcio y magnesio en el complejo de cambio, originando entre otras cosas, pérdida de la estructura en el suelo haciéndolo impermeable (Sánchez, 2006).

\section{Conclusion}

Con base a los resultados obtenidos se concluye, que en los sitios estudiados donde se siembra el cultivo del espárrago, se observó que la fertilización con nitrógeno y potasio al suelo se realiza en exceso, lo contrario fue detectado con el elemento fósforo, tomando como base los análisis de referencia de los laboratorios. Los resultados obtenidos también confirman que la realización del análisis de suelo forma parte de una herramienta integral de diagnóstico nutrimental que cuando se lleva a cabo un análisis únicamente de la parte foliar o de suelo de forma separada, el diagnóstico para el cultivo de espárrago por lo tanto resultó incompleto, lo que implica que no se aseguren aplicaciones de las dosis realmente requeridas para el cultivo, excediéndose o aplicando menor cantidad. Es necesario la realización de más estudios sobre el ciclo de desarrollo del espárrago para la región agrícola de Caborca, Sonora, a efecto de poder correlacionar con mayor precisión, los estudios con en relación a los requerimientos nutricionales del cultivo, debido a que la aplicación de fertilizantes en exceso eleva los costos de producción y además, al lixiviarse provoca contaminación de los suelos y mantos freáticos.

\section{References:}

1. Aceves, N. E. Ensalitramiento de suelos bajo riego. Ed. Colegio de Posgraduados Rama de Riego y Drenaje. Chapingo México. 1979. 382 p. 
2. Aguilar, S. A.; Etchevers, B. J.; Castellanos J. Z. Análisis químico para evaluar la fertilidad del suelo. Sociedad Mexicana de la Ciencia del Suelo. Publicación Especial. 1987. No 1217 p.

3. Cabrera, Y. V. Análisis de los factores de producción en espárrago en la Pampa de Villacuri. Tesis. Universidad Nacional Agraria La Molina. Perú (Ica, Perú). 1993.173 p.

4. De Santa Olalla, M. F.; López F. P.; Calera B. Agua y Agronomía. Editorial Mundi-Prensa. España. 2005. 330 p.

5. Espejo, J. A.; Tejada M.; Benitez C.; González, J.L. 1997. Change in $\mathrm{pH}$ and the organic matter and phosphorus contents of asparagus cultivated soils produced by phosphorus fertilization. Journal of Plant Nutrition 20 (11): 1465-1478.

6. Ferreyra, E. R.; Sellés Van G.; Peralta, S., J. M.; Fritsch F N.; Contador, F.; Rubio F. Respuesta del Cultivo del Espárrago (Asparagus officinalis L.) a Distintos Regímenes de Riego Durante las Dos Primeras Temporadas de Establecimiento. Agricultura Técnica. Chile. 1995.55 (1): 1-8.

7. Francois, L. E. Salinity effects on Asparagus yield and vegetative growth. J Amer. Soc. Hort. Sci. 1987. 112 (3):432-436

8. Gardner, B. R.; Roth, R. L. Plant analysis for nitrogen fertilization of asparagus. J. Amer. Soc. Hort Sci. 1989. 114:741-745

9. Licon, T. L. P.; Rodríguez, V. L.; Molina, J. N. L. Diagnóstico, Interpretación y Resultados. Manual de Análisis de Suelos-PlantaAgua. Universidad Autónoma de Chiapas. 2001. pp 9-14.

10. Montes, A.; Holle, M. El cultivo de espárrago en el Trópico. Programa de investigación en Hortalizas. UANLM. Facultad de Agronomía. Lima Perú. 1978. 46 p.

11. Mullen, R.; Laemlen F. F. Asparagus Production in California. University of California. Division of Agriculture and Natural Resources. Publication 7234. 2008. pp. 1-3

12. Navarro, A. J. A. Manejo de la Fertilización y el Riego. Rev. Tecnología para el Manejo del Espárrago en el Noroeste de Sonora. SAGARPA-INIFAP. Caborca, Son., México. 2002. No. 6: 31p.

13. Núñez, G. E.; Casas A. D. Composición Nutricional de Turiones de Espárrago Verde (Asparagus officinalis L.) y su Relación con la Vida Post-Cosecha. I Congreso Nacional Técnico del Esparrago. LimaPerú. 2000. 8 p.

14. López-Aguilar, R.; Murillo-Amador, B.; Rodríguez-Quezada, G. El forraje verde hidropónico (FVH): Una alternativa de producción de alimento para el ganado en zonas áridas. Interciencia. 2008. 34 (2): 121-126. 
15. Orduño, Z. J. A. Nutrición en espárrago. Memorias del Curso de capacitación sobre programas de inocuidad y $2^{\circ}$ Seminario en espárrago. Comité Estatal de Sanidad Vegetal de Sonora. Caborca, Sonora. México. 2004. 72 p.

16. Pérez, R. V.; Werner R. C. Apuntes del Curso de Desarrollo de Técnicas de Fertilidad y Salinidad del suelo y perfil fisicoquímico de fertilizantes orgánicos e inorgánicos. CIAD Unidad Culiacán, Sin., México. 2007. 54 p.

17. Porta, J.; López-Acevedo, M.; Roguero, D. C. Edafología para la Agricultura y el medio ambiente. Ed. Mundi-Prensa. Madrid, España. 1999. $871 \mathrm{p}$.

18. Rueda-Puente, E. O.; Barrón-Hoyos, J.; Tarazón-Herrera, M.; Preciado-Rangel, P.; La Salinidad: Un Problema o Una Opción Para La Agricultura. Editorial Plaza y Valdes. México D.F. 2009. 264 p.

19. Rueda-Puente E, Castellanos T, Troyo-Diéguez E, Díaz de LeónÁlvarez J, Murillo-Amador B. Effects of a nitrogen-fixing indigenous bacterium Klebsiella pneumoniae on the growth and development of the halophyte Salicornia bigelovii as a new crop for saline environments. Journal Agronomy of Crops Sciences. 2003. 189: 323-332.

20. Rueda-Puente, E. O.; Barrón-Hoyos, J.; Tarazón-Herrera, M.; Preciado-Rangel, P.; La Salinidad: Un Problema o Una Opción Para La Agricultura. Editorial Plaza y Valdes. México D.F. 2009. 264 p.

21. Ruíz, B. A.; Ortega T. E. Prácticas de Laboratorio. Química de Suelos. Universidad Autónoma de Chapíngo. Texcoco, Estado de México. 1979. 76 p.

22. Sánchez, V. J. Nutrición y Fertilización del Cultivo de Espárrago. Conferencia dictada en: Modulo Integrado de Espárrago y Alcachofas. Facultad de Agronomía. UNALM. Lima Perú. 2005. 19 p.

23. Sánchez, V. J. Fertirrigación en el cultivo del espárrago en Perú. En: Fertirrigacao: Teoría y Práctica. A. E. Boaretto, R. L. Villas Boas, V. S. Sousa e I. R. Vidal. Universidad de Sao Paulo. Piracicaba, Sao Paulo, Brasil. 2006. 22 p.

24. Santelises, A. A.; Etchevers B. J. D.; Castellanos, R. J. Z. Análisis Químico para Evaluar la Fertilidad del Suelo. Sociedad Mexicana de las Ciencias del Suelo. Publicación Especial. No.1. 1987. 217 p.

25. SARH. Secretaría de Agricultura y Recursos Hidráulicos. Programa Distrital de Desarrollo Rural Integral. 1987-1992. Resumen. México. 1987. 28 p.

26. SAGARPA. Secretaría de Agricultura, Ganadería, Desarrollo Rural, Pesca y Alimentación, Análisis Agropecuario del Tomate. Boletín 
Informativo. Culiacán, Sinaloa, México. 2005. 9 p.Souther, F.; Benson, B. L.; Bravo, A. Tecnología de producción de espárrago. Ed. Chile. Chile. 1986. 288 p.

\section{INDICE DE CUADROS}

Cuadro 1.Sitios de estudio, subregión y edad de plantaciones de espárrago en Caborca, Sonora.

Cuadro 2. Análisis fisicoquímicos realizados en suelo y métodos de laboratorio utilizados.

Cuadro 3. Estándares de referencia de los laboratorios de suelos en México. Cuadro 4. Análisis fisicoquimícos de suelo analizados donde se siembra cultivo de Espárrago. Sitio de Estudio I

Cuadro 5. Análisis fisicoquimícos de suelo analizados donde se siembra cultivo de Espárrago. Sitio de Estudio II

Cuadro 6. Análisis fisicoquimícos de suelo analizados donde se siembra cultivo de Espárrago. Sitio de Estudio III

Cuadro 7. Análisis fisicoquimícos de suelo analizados donde se siembra cultivo de Espárrago. Sitio de Estudio IV

Cuadro 8. Análisis fisicoquimícos de suelo analizados donde se siembra cultivo de Espárrago. Sitio de Estudio V

Cuadro 9. Análisis fisicoquimícos de suelo analizados donde se siembra cultivo de Espárrago. Sitio de Estudio VI

Cuadro 10. Análisis fisicoquimícos de suelo analizados donde se siembra cultivo de Espárrago. Sitio de Estudio VII

Cuadro 1. Sitios de estudio, subregión y edad de plantaciones de espárrago en Caborca, Sonora.

\begin{tabular}{ccc}
\hline Sitio Experimental & Subregión & $\begin{array}{c}\text { Edad } \\
\text { (Años) }\end{array}$ \\
\hline Sitio I & Pitiquito & 14 \\
Sitio II & La Victoria & 13 \\
Sitio III & El Bizani & 11 \\
Sitio IV & Los Sapos & 12 \\
Sitio V & La Y & 19 \\
Sitio VI & La Y & 13 \\
Sitio VII & Enchilayas & 11 \\
\hline
\end{tabular}


Cuadro 2. Análisis fisicoquímicos realizados en suelo y métodos de laboratorio utilizados.

\begin{tabular}{ll}
\hline Análisis Fisico- Quimico & Método de Laboratorio Utilizado \\
\hline Nitrógeno $\left(\mathrm{NO}_{3}\right)$ & Método de Acido Fenoldisulfónico \\
Fósforo & Método Bray P-1 \\
Potasio & Método Acetato Amonio 1 N. pH8 \\
Calcio & Método Acetato Amonio 1 N. pH8 \\
Magnesio & Método Acetato Amonio 1 N. pH8 \\
Sodio & Método Acetato Amonio 1 N. pH8 \\
Textura & Hidrómetro de Bouyoucos \\
Ph & En pasta saturada \\
Conductividad eléctrica & CE en Extracto de saturación \\
\% Saturación & Pasta saturada \\
\hline
\end{tabular}

Cuadro 3. Estándares de referencia de los laboratorios de suelos en México.

\begin{tabular}{cc}
\hline Nutriente & Niveles adecuados (ppm) \\
\hline $\mathrm{NO}_{3}$ & 30 \\
$\mathrm{P}$ & 30 \\
$\mathrm{~K}$ & 350 \\
$\mathrm{Ca}$ & 2000 \\
$\mathrm{Mg}$ & 250 \\
$\mathrm{Na}$ & $<250$ \\
\hline
\end{tabular}

Cuadro 4. Análisis fisicoquimícos de suelo analizados donde se siembra cultivo de Espárrago. Sitio de Estudio I

\begin{tabular}{ccccccccccc}
\hline MES & \% SAT. & $\mathrm{pH}$ & $\begin{array}{c}\text { C.E. } \\
(\mathrm{mmhos})\end{array}$ & $\begin{array}{c}\mathrm{NO}_{3} \\
(\mathrm{ppm})\end{array}$ & $\begin{array}{c}\mathrm{P} \\
(\mathrm{ppm})\end{array}$ & $\begin{array}{c}\mathrm{Ca} \\
(\mathrm{ppm})\end{array}$ & $\begin{array}{c}\mathrm{Mg} \\
(\mathrm{ppm})\end{array}$ & $\begin{array}{c}\mathrm{Na} \\
(\mathrm{ppm})\end{array}$ & $\begin{array}{c}\mathrm{K} \\
(\mathrm{ppm})\end{array}$ & $\begin{array}{c}\text { C.I.C. } \\
(\mathrm{mEq} / 100 \mathrm{gm})\end{array}$ \\
\hline MARZO & 30.6 & 7.0 & 5.0 & 46.6 & 4.6 & 2203 & 161 & 634 & 1254 & 22.8 \\
ABRIL & 30.4 & 7.2 & 5.9 & 36.1 & 13.9 & 3197 & 120 & 566 & 1104 & 22.2 \\
MAYO & 28 & 7.2 & 7.2 & 19.6 & 22.5 & 3865 & 606 & 1497 & 1941 & 30.8 \\
SEPTIEMBRE & 18 & 7.2 & 9.2 & 47.5 & 10.2 & 2219 & 477 & 729 & 784 & 20.1 \\
OCTUBRE & 17.5 & 7.1 & 2.1 & 39.3 & 11.6 & 2301 & 278 & 491 & 977 & 18.4 \\
NOVIEMBRE & 17.7 & 7.2 & 5.7 & 71.0 & 62.0 & 1940 & 590 & 2439 & 780 & 27.1 \\
DICIEMBRE & 23.2 & 7.4 & 12.1 & 10.5 & 6.1 & 2115 & 491 & 2515 & 561 & 27.0 \\
FEBRERO & 27.2 & 7.4 & 13.1 & 73.0 & 4.1 & 2130 & 570 & 1836 & 2646 & 30.1 \\
MARZO & 16.8 & 7.5 & 4.7 & 30.0 & 10.8 & 2043 & 523 & 2185 & 332 & 25.1 \\
ABRIL & 27.13 & 7.4 & 5.3 & 42.0 & 15.5 & 654 & 442 & 2271 & 361 & 17.6 \\
\hline
\end{tabular}


Cuadro 5. Análisis fisicoquimícos de suelo analizados donde se siembra cultivo de Espárrago. Sitio de Estudio II

\begin{tabular}{ccccccccccc}
\hline MES & \% SAT. & pH & $\begin{array}{c}\text { C.E. } \\
(\mathrm{mmhos})\end{array}$ & $\begin{array}{c}\mathrm{NO}_{3} \\
(\mathrm{ppm})\end{array}$ & $\begin{array}{c}\mathrm{P} \\
(\mathrm{ppm})\end{array}$ & $\begin{array}{c}\mathrm{Ca} \\
(\mathrm{ppm})\end{array}$ & $\begin{array}{c}\mathrm{Mg} \\
(\mathrm{ppm})\end{array}$ & $\begin{array}{c}\mathrm{Na} \\
(\mathrm{ppm})\end{array}$ & $\begin{array}{c}\mathrm{K} \\
(\mathrm{ppm})\end{array}$ & $\begin{array}{c}\text { C.I.C. } \\
(\mathrm{mEq} / 100 \mathrm{gm})\end{array}$ \\
\hline MARZO & 30.2 & 7.2 & 5.9 & 52.1 & 34.8 & 2405 & 125 & 705 & 1711 & 20.1 \\
ABRIL & 31.6 & 7.3 & 2.7 & 40.3 & 12.5 & 2707 & 74 & 467 & 287 & 16.8 \\
MAYO & 26.5 & 7.2 & 7.7 & 74.0 & 26.7 & 3174 & 477 & 1933 & 3054 & 35.8 \\
SEPTIEMBRE & 21.6 & 7.6 & 4.0 & 21.1 & 10.9 & 1726 & 205 & 298 & 900 & 13.9 \\
OCTUBRE & 19.5 & 7.2 & 6.0 & 83.3 & 20.4 & 1820 & 190 & 681 & 1494 & 17.4 \\
NOVIEMBRE & 21.4 & 7.2 & 5.8 & 88.3 & 33.0 & 1784 & 396 & 1959 & 868 & 22.9 \\
DICIEMBRE & 29.6 & 7.4 & 7.8 & 35.2 & 5.6 & 2045 & 420 & 2421 & 1188 & 27.2 \\
FEBRERO & 30 & 7.6 & 6.7 & 100.3 & 24.7 & 1769 & 437 & 1800 & 2553 & 26.3 \\
MARZO & 27 & 7.6 & 3.7 & 50.0 & 31.0 & 1862 & 369 & 2034 & 443 & 22.3 \\
ABRIL & 25 & 7.6 & 4.6 & 39.3 & 14.0 & 559 & 382 & 2338 & 560 & 17.5 \\
\hline
\end{tabular}

Cuadro 6. Análisis fisicoquimícos de suelo analizados donde se siembra cultivo de Espárrago. Sitio de Estudio III

\begin{tabular}{ccccccccccc}
\hline MES & \% SAT. & $\mathrm{pH}$ & $\begin{array}{c}\text { C.E. } \\
(\mathrm{mmhos})\end{array}$ & $\begin{array}{c}\mathrm{NO}_{3} \\
(\mathrm{ppm})\end{array}$ & $\begin{array}{c}\mathrm{P} \\
(\mathrm{ppm})\end{array}$ & $\begin{array}{c}\mathrm{Ca} \\
(\mathrm{ppm})\end{array}$ & $\begin{array}{c}\mathrm{Mg} \\
(\mathrm{ppm})\end{array}$ & $\begin{array}{c}\mathrm{Na} \\
(\mathrm{ppm})\end{array}$ & $\begin{array}{c}\mathrm{K} \\
(\mathrm{ppm})\end{array}$ & $\begin{array}{c}\text { C.I.C. } \\
(\mathrm{mEq} / 100 \mathrm{gm})\end{array}$ \\
\hline MARZO & 28.1 & 7.3 & 1.1 & 12.5 & 31.1 & 881 & 17 & 398 & 195 & 6.7 \\
ABRIL & 22.1 & 7.1 & 3.5 & 25.1 & 23.6 & 1802 & 32 & 378 & 528 & 12.2 \\
MAYO & 26.4 & 7.5 & 1.9 & 30.0 & 17.9 & 1045 & 287 & 922 & 188 & 12.0 \\
SEPTIEMBRE & 22 & 7.7 & 1.0 & 9.6 & 31.7 & 1345 & 213 & 1781 & 32 & 16.3 \\
OCTUBRE & 18.8 & 6.9 & 1.2 & 29.8 & 16.7 & 1410 & 100 & 482 & 0 & 9.9 \\
NOVIEMBRE & 24.2 & 2.1 & 1.1 & 29.0 & 37.0 & 1294 & 274 & 1910 & 414 & 18.3 \\
DICIEMBRE & 22 & 7.3 & 2.1 & 23.8 & 22.3 & 1395 & 252 & 2104 & 109 & 18.4 \\
FEBRERO & 27 & 7.6 & 0.9 & 30.0 & 26.0 & 1365 & 239 & 1586 & 607 & 17.2 \\
MARZO & 28.6 & 7.2 & 1.3 & 28.6 & 38.3 & 1367 & 270 & 1868 & 229 & 17.8 \\
ABRIL & 26.6 & 7.3 & 1.4 & 17.8 & 4.14 & 357 & 217 & 2096 & 148 & 13.0 \\
\hline
\end{tabular}

Cuadro 7. Análisis fisicoquimícos de suelo analizados donde se siembra cultivo de Espárrago. Sitio de Estudio IV

\begin{tabular}{ccccccccccc}
\hline MES & \% SAT. & $\mathrm{pH}$ & $\begin{array}{c}\mathrm{C} . \mathrm{E} . \\
(\mathrm{mmhos})\end{array}$ & $\begin{array}{c}\mathrm{NO}_{3} \\
(\mathrm{ppm})\end{array}$ & $\begin{array}{c}\mathrm{P} \\
(\mathrm{ppm})\end{array}$ & $\begin{array}{c}\mathrm{Ca} \\
(\mathrm{ppm})\end{array}$ & $\begin{array}{c}\mathrm{Mg} \\
(\mathrm{ppm})\end{array}$ & $\begin{array}{c}\mathrm{Na} \\
(\mathrm{ppm})\end{array}$ & $\begin{array}{c}\mathrm{K} \\
(\mathrm{ppm})\end{array}$ & $\begin{array}{c}\text { C.I.C. } \\
(\mathrm{mEq} / 100 \mathrm{gm})\end{array}$ \\
\hline MARZO & 16.6 & 7.4 & 5.3 & 3.0 & 18.3 & 1213 & 61 & 709 & 206 & 10.1 \\
ABRIL & 16.9 & 7.6 & 7.6 & 3.1 & 18.0 & 1577 & 52 & 509 & 440 & 11.6 \\
MAYO & 15.3 & 7.3 & 7.0 & 2.8 & 37.5 & 1031 & 446 & 1281 & 871 & 14.1 \\
SEPTIEMBRE & 9.9 & 7.6 & 3.9 & 5.8 & 11.9 & 1864 & 227 & 344 & 951 & 15.1 \\
OCTUBRE & 12.3 & 7.3 & 4.8 & 14.5 & 28.8 & 1819 & 148 & 658 & 354 & 14.0 \\
NOVIEMBRE & 18.1 & 7.5 & 6.9 & 40.8 & 34.0 & 1507 & 341 & 1957 & 265 & 19.5 \\
DICIEMBRE & 16 & 7.6 & 7.5 & 7.3 & 38.1 & 1752 & 379 & 2297 & 555 & 23.2 \\
FEBRERO & 16 & 7.6 & 7.5 & 7.3 & 38.1 & 1752 & 379 & 2297 & 555 & 23.2 \\
MARZO & 15.8 & 7.6 & 3.7 & 10.0 & 27.9 & 1553 & 444 & 2168 & 153 & 21.2 \\
ABRIL & 16.8 & 7.7 & 4.4 & 14.8 & 20.8 & 474 & 303 & 2433 & 236 & 16.0
\end{tabular}


Cuadro 8. Análisis fisicoquimícos de suelo analizados donde se siembra cultivo de Espárrago. Sitio de Estudio V

\begin{tabular}{ccccccccccc}
\hline MES & \% SAT. & $\mathrm{pH}$ & $\begin{array}{c}\text { C.E. } \\
(\mathrm{mmhos})\end{array}$ & $\begin{array}{c}\mathrm{NO}_{3} \\
(\mathrm{ppm})\end{array}$ & $\begin{array}{c}\mathrm{P} \\
(\mathrm{ppm})\end{array}$ & $\begin{array}{c}\mathrm{Ca} \\
(\mathrm{ppm})\end{array}$ & $\begin{array}{c}\mathrm{Mg} \\
(\mathrm{ppm})\end{array}$ & $\begin{array}{c}\mathrm{Na} \\
(\mathrm{ppm})\end{array}$ & $\begin{array}{c}\mathrm{K} \\
(\mathrm{ppm})\end{array}$ & $\begin{array}{c}\text { C.I.C. } \\
(\mathrm{mEq} / 100 \mathrm{gm})\end{array}$ \\
\hline MARZO & 20.8 & 7.2 & 5.5 & 29.5 & 59.8 & 1273 & 147 & 662 & 627 & 11.0 \\
ABRIL & 19.8 & 7.1 & 8.8 & 38.3 & 28.6 & 1775 & 80 & 437 & 623 & 13.0 \\
MAYO & 19.2 & 7.3 & 4.9 & 26.0 & 31.2 & 1719 & 449 & 2215 & 1743 & 29.7 \\
SEPTIEMBRE & 16.4 & 7.6 & 6.5 & 26.0 & 27.8 & 1456 & 266 & 313 & 443 & 11.9 \\
OCTUBRE & 18.7 & 7.1 & 7 & 73.6 & 19.4 & 1615 & 139 & 640 & 670 & 13.6 \\
NOVIEMBRE & 21.1 & 7.4 & 4.4 & 36.0 & 51.0 & 1367 & 440 & 1997 & 502 & 20.4 \\
DICIEMBRE & 26.6 & 7.6 & 4.8 & 16.6 & 35.1 & 1484 & 434 & 2312 & 732 & 22.9 \\
FEBRERO & 23 & 7.4 & 5.2 & 40.6 & 36.3 & 1472 & 438 & 1689 & 1662 & 22.5 \\
MARZO & 21.3 & 7.6 & 6.3 & 51.6 & 29.3 & 1465 & 422 & 2142 & 531 & 22.1 \\
ABRIL & 21.13 & 7.61 & 5.86 & 29.3 & 23.65 & 357 & 418 & 2302 & 680 & 17.0 \\
\hline
\end{tabular}

Cuadro 9. Análisis fisicoquimícos de suelo analizados donde se siembra cultivo de Espárrago. Sitio de Estudio VI

\begin{tabular}{ccccccccccc}
\hline MES & \% SAT. & $\mathrm{pH}$ & $\begin{array}{c}\text { C.E. } \\
(\mathrm{mmhos})\end{array}$ & $\begin{array}{c}\mathrm{NO}_{3} \\
(\mathrm{ppm})\end{array}$ & $\begin{array}{c}\mathrm{P} \\
(\mathrm{ppm})\end{array}$ & $\begin{array}{c}\mathrm{Ca} \\
(\mathrm{ppm})\end{array}$ & $\begin{array}{c}\mathrm{Mg} \\
(\mathrm{ppm})\end{array}$ & $\begin{array}{c}\mathrm{Na} \\
(\mathrm{ppm})\end{array}$ & $\begin{array}{c}\mathrm{K} \\
(\mathrm{ppm})\end{array}$ & $\begin{array}{c}\text { C.I.C. } \\
(\mathrm{mEq} / 100 \mathrm{gm})\end{array}$ \\
\hline MARZO & 20.8 & 7.3 & 11.36 & 31.8 & 14.1 & 2915 & 208 & 837 & 1194 & 22.9 \\
ABRIL & 20.2 & 7.1 & 18.2 & 53.0 & 26.6 & 2755 & 90 & 667 & 1274 & 20.5 \\
MAYO & 16.5 & 7 & 15.5 & 33.1 & 37.1 & 1981 & 391 & 1369 & 1126 & 21.9 \\
SEPTIEMBRE & 15.7 & 7.5 & 2.4 & 61.0 & 10.3 & 2138 & 298 & 529 & 1069 & 18.2 \\
OCTUBRE & 14.6 & 7 & 5 & 50.0 & 29.2 & 2210 & 222 & 654 & 801 & 17.6 \\
NOVIEMBRE & 20.5 & 7.2 & 5.5 & 42.0 & 51.0 & 1681 & 332 & 1847 & 517 & 20.5 \\
DICIEMBRE & 16.6 & 7.5 & 10.1 & 38.3 & 25.5 & 2001 & 326 & 2227 & 862 & 24.5 \\
FEBRERO & 20 & 7.1 & 14.4 & 60.0 & 21.0 & 1674 & 389 & 1831 & 1776 & 24.1 \\
MARZO & 16.8 & 7.5 & 8.4 & 46.0 & 9.0 & 2106 & 408 & 2127 & 463 & 24.3 \\
ABRIL & 17.7 & 7.24 & 6.33 & 23.5 & 26.33 & 520 & 266 & 2310 & 289 & 15.6 \\
\hline
\end{tabular}

Cuadro 10. Análisis fisicoquimícos de suelo analizados donde se siembra cultivo de Espárrago. Sitio de Estudio VII

\begin{tabular}{ccccccccccc}
\hline MES & \% SAT. & $\mathrm{pH}$ & $\begin{array}{c}\text { C.E. } \\
(\mathrm{mmhos})\end{array}$ & $\begin{array}{c}\mathrm{NO}_{3} \\
(\mathrm{ppm})\end{array}$ & $\begin{array}{c}\mathrm{P} \\
(\mathrm{ppm})\end{array}$ & $\begin{array}{c}\mathrm{Ca} \\
(\mathrm{ppm})\end{array}$ & $\begin{array}{c}\mathrm{Mg} \\
(\mathrm{ppm})\end{array}$ & $\begin{array}{c}\mathrm{Na} \\
(\mathrm{ppm})\end{array}$ & $\begin{array}{c}\mathrm{K} \\
(\mathrm{ppm})\end{array}$ & $\begin{array}{c}\text { C.I.C. } \\
(\mathrm{mEq} / 100 \mathrm{gm})\end{array}$ \\
\hline MARZO & 20.2 & 7.2 & 2 & 8.0 & 52.6 & 1428 & 117 & 513 & 314 & 11.1 \\
ABRIL & 19.2 & 7.3 & 2 & 6.8 & 27.0 & 1465 & 78 & 439 & 91.3 & 10.0 \\
MAYO & 17.1 & 7.1 & 2.6 & 18.5 & 53.6 & 2047 & 457 & 684 & 814 & 28.8 \\
SEPTIEMBRE & 12.5 & 7.5 & 5.1 & 49.8 & 25.4 & 1589 & 251 & 386 & 631 & 13.3 \\
OCTUBRE & 14.5 & 7.2 & 2.8 & 56.8 & 22.9 & 1869 & 153 & 674 & 551 & 14.9 \\
NOVIEMBRE & 20.5 & 7.6 & 3 & 47.1 & 23.0 & 1595 & 354 & 2002 & 484 & 20.8 \\
DICIEMBRE & 17.4 & 7.2 & 8.4 & 42.0 & 40.6 & 1669 & 3883 & 2322 & 807 & 23.7 \\
FEBRERO & 18 & 7.3 & 3.4 & 39.3 & 17.4 & 1762 & 435 & 1787 & 349 & 21.2 \\
MARZO & 17.2 & 7.5 & 5.3 & 34.6 & 29.4 & 1596 & 366 & 1992 & 335 & 20.6 \\
ABRIL & 18.2 & 7.45 & 2.7 & 32.1 & 21.8 & 449 & 374 & 2299 & 198 & 15.8 \\
\hline
\end{tabular}

